\title{
Les chauves-souris cavernicoles de la Suisse
}

\author{
Par Villy Aellen ${ }^{1}$ ) \\ Avec planches 67 (1) - 69 (3)
}

Chacun sait que les chauves-souris jouent un rôle important dans la spéléologie en général et dans la faune cavernicole en particulier.

Nombreux, en effet, sont les clubs qui ont adopté son image plus ou moins stylisée comme emblème. Mais cette symbolisation n'est pas le moindre mérite de ces animaux que l'on souhaiterait voir aimés de chacun. Reconnues très utiles en tant qu'insectivores dans tous les milieux, sauf celui des agriculteurs qui auraient pourtant tout à gagner en les protégeant, elles ont rendu souvent d'appréciables services aux spéléologues, dans des domaines très divers. Leur présence rassurante a permis à maint jeune homme de trouver le courage nécessaire pour continuer son exploration solitaire. Parfois même, elles ont légitimé l'existence d'une autre sortie, lorsqu'elles ont été observées au-delà d'une voûte mouillante ou après le creusage à la barre à mine d'une galerie terminale.

Dans le domaine de la faune cavernicole qui nous occupe présentement, les chauves-souris occupent une place importante. Non seulement leur présence même doit être notée, comme celle de tout animal rencontré sous terre, mais encore leurs déjections composent un milieu extrêmement favorable à toute une faune particulière. Les animaux vivant dans ce guano ne se trouvent en effet que très rarement ailleurs et ces guanobies représentent un élément essentiel et caractéristique de la faune cavernicole. Quelques exemples permetteront de mieux comprendre le rôle prépondérant joué indirectement par les chauvessouris dans le peuplement animal des grottes. Un coléoptère staphylinide, Quedius mesomelinus, est présent dans la plupart des grottes renfermant des déjections de chauves-souris, même s'il n'y a que quelques crottes éparses, et cela dans la plus grande partie de l'Europe. Mais en dehors de ces grottes à guano, le staphylin est très rare. Un autre exemple est encore plus caractéristique, quoique moins connu. Le collembole Mesachorutes ojcoviensis, trouvé d'abord dans une grotte

1) Muséum d'Histoire Naturelle, Genève, Suisse. 
de Pologne, a ensuite été observé, toujours dans des grottes, dans les Carpathes méridionales, au Tyrol autrichien, dans le nord et le sud de l'Italie, en Espagne et enfin dans une grotte du Jura neuchâtelois. Dans chaque cas, il s'agit de grottes renfermant du guano. La seule trouvaille faite en dehors de grottes concerne des nids de petits rongeurs dans les Pyrénées; mais là aussi le collembole vivait dans les crottes de ces campagnols.

Un apport renouvelé de guano et l'accumulation considérable de celui-ci augmentent sensiblement la température - et par conséquence l'humidité absolue - d'une grotte, grâce à la fermentation des matières organiques. Ces conditions particulières créent un micro-climat avantageux non seulement pour les guanobies spécialisés, mais aussi pour toute une foule d'autres animaux cavernicoles qui trouvent là un milieu physique moins rigoureux favorisant leur multiplication.

Un autre apport indirect des chauves-souris à la faune cavernicole est celui de leurs parasites. On sait que les tiques (Ixodes), les puces et les nyctéribies (diptères sans ailes) passent au cours de leur développement par une phase libre; ils errent alors sur les parois des grottes à la recherche d'un hôte d'élection.

La répartition classique des animaux cavernicoles dans les trois catégories des troglobies, troglophiles et trogloxènes, s'applique difficilement aux chauves-souris. Aussi, sont-elles traitées généralement à part dans les listes de faune spéléologique. Vingt-six espèces de chauves-souris habitent la Suisse, mais quinze seulement se rencontrent plus ou moins régulièrement dans notre domaine souterrain. Une seule, le minioptère, peut être qualifiée de vraie cavernicole; elle ne s'abrite en effet que dans les grottes et dans les souterrains artificiels. Dans une deuxième catégorie, on peut placer les espèces qui fréquentent régulièrement les grottes, au moins en hiver, mais qui se retirent aussi en d'autres lieux, notamment pour la mise-bas; ce sont les rhinolophes, quelques espèces de murins, la barbastelle et l'oreillard. Enfin un dernier groupe renferme les chauves-souris, en assez grand nombre au point de vue qualitatif, qui se trouvent occasionnellement dans les grottes, surtout pendant la mauvaise saison. Il s'agit soit d'espèces arboricoles, souvent rares (murins de Bechstein, de Natterer), soit d'espèces localisées dans certaines régions de notre pays (sérotine boréale, murin de Capaccini).

\section{Miniopterus schreibersi (Kuhl)}

Le minioptère est une espèce méridionale atteignant en Suisse la limite nord de son aire de répartition. C'est la seule de nos chauvessouris qui se rassemble en colonies populeuses, pouvant compter 
plusieurs milliers d'individus agglomérés en essaim. Habitant strictement les grottes naturelles ou artificielles, il a d'abord été observé dans la grotte de Môtiers (NE $8^{2}$ ) il y a un siècle. La colonie de cette grotte, prospère autrefois, a maintenant disparu et on ne l'y observe plus qu'irrégulièrement en de rares individus.

Il y a une vingtaine d'années, une petite colonie avait élu domicile dans les caves du Bois de la Bâtie, à Genève; mais elle a disparu complètement depuis plusieurs années. Une autre colonie a fréquenté temporairement la Beatushöhle (BE 16), au moins en automne 1952; F. Knuchel y a bagué 65 minioptères, mais depuis lors la colonie s'est mystérieusement évanouie; on n'en a pas relevé la moindre trace, aucun individu n'ayant été retrouvé ailleurs.

Les seules grottes habitées actuellement d'une façon assez régulière sont la grotte du Poteux (VS 2) au Valais et celle du Chemin de Fer (NE 14) dans le Jura neuchâtelois. Environ 250 minioptères ont été bagués par M. Desfayes dans la première et plus de 1000 dans celle du Chemin de Fer (y compris la grotte de Ver, voisine), d'abord par H. Mislin, puis par moi-même et quelques collaborateurs. De très nombreuses reprises ont été faites en Suisse et en France, qui permettent de préciser quelque peu les déplacements de nos minioptères. Un fait semble acquis: aucune de nos grottes ne sert de lieu de mise-bas; cette opération se fait dans des cavernes du Jura français. Des échanges ont lieu continuellement entre les deux colonies de la Suisse et celles du Jura français. De nombreuses trouvailles d'individus isolés ou en petit nombre ont été faites entre le Jura et le Valais, soit dans des grottes, soit fortuitement près de lieux habités ou sur la route (animal tué par une automobile, par exemple). Ce n'est pas le lieu ici d'analyser en détail ces captures. Disons seulement que des minioptères ont été trouvés dans quinze lieux différents en Suisse Romande, dont sept sont des grottes porfois insignifiantes et ne servant que d'abri temporaire aux chauves-souris en migration (planche 1).

\section{Rhinolophus hipposideros (Bechstein)}

Le rhinolophe petit fer à cheval est l'espèce la plus fréquemment rencontrée sous terre. Il s'y trouve généralement en individus isolés, mais parfois aussi en colonies groupant jusqu'à quelques dizaines d'individus. Il se tient suspendu à la voûte ou à une aspérité uniquement par ses pattes postérieures, la tête en bas, comme toutes les chauves-souris, dans une attitude très caractéristique, les ailes enveloppant tout le corps et le faisant ressembler à un fruit pendant.

2) Cf. Aellen et Strinati (1962). 
On l'a déjà trouvé dans 80 grottes environ du Jura, du Plateau, des Alpes et du Tessin, principalement en hiver. Pendant la belle saison, il peut se contenter d'un refuge très précaire: un simple abri sous roche ou une faille étroite. En dehors des grottes, il est observé souvent dans les combles ou les caves des églises et des vieilles bâtisses, à la condition qu'il n'y soit pas dérangé trop souvent.

\section{Rhinolophus ferrumequinum (Schreber)}

De mœurs semblables à celles du petit fer à cheval, le grand rhinolophe est plus rare dans notre pays, sauf peut-être au Tessin. Il monte moins haut dans les Alpes et le Jura. On l'a trouvé jusqu'à présent dans 45 grottes. Au repos, il s'enveloppe moins complètement dans ses ailes que Rh.hipposideros.

Nous n'avons jamais constaté en Suisse de grotte-maternité pour nos deux espèces de rhinolophes.

\section{Myotis myotis (Borkhausen)}

Nous ne connaissons pas en Suisse de grottes renfermant des colonies de grands murins, comme cela se trouve dans le Jura français, par exemple. Les individus observés chez nous sous terre sont toujours des isolés ou des couples en hiver. Pendant la belle saison, le grand murin s'abrite volontiers sous les combles ou dans les tours des églises où les femelles se réunissent en colonies populeuses en mai ou juin pour la mise-bas.

Une cinquantaine de grotte de notre pays sont connues pour servir de refuge de temps à autre au grand murin. Les nombreux individus bagués en hiver dans les grottes n'ont jamais été retrouvés dans des colonies estivales hors de grottes; et vice versa, aucun murin, parmi les centaines bagués en été dans les combles, n'a été repris en hiver sous terre. Il est certain que nos grands murins entreprennent des déplacements saisonniers, comme cela a été constaté hors de nos frontières, mais on n'en connaît pas du tout les modalités.

\section{Myotis mystacinus (Kuhl)}

Un peu moins fréquent sous terre que l'espèce précédente, le murin à moustaches a été trouvé dans 28 grottes du Jura et des Alpes. De taille minuscule, il est fréquemment confondu avec la pipistrelle. On ne le trouve guère dans nos grottes qu'en hiver et toujours isolé, souvent profondément enfoncé dans une fissure de la voûte. En été, il doit se contenter d'un trou d'arbre ou d'une fente de rocher. Les 
femelles se rassemblent à la fin de mai en petites colonies sous des toits ou sous les combles où elles mettent bas.

\section{Barbastella barbastellus (Schreber)}

La barbastelle est largement répandue en Suisse, bien qu'assez rarement trouvée hors des grottes. Elle s'est rencontrée dans 22 cavernes du Jura et des Alpes. D'habitude, elle s'y trouve en hiver isolée ou par couple dans une encoignure ou dans une fissure, parfois très près de l'entrée, à côté de glaçons. Mais il arrive quelquefois que des dizaines de barbastelles se rassemblent et constituent un véritable essaim, comme les minioptères. J'ai observé à diverses reprises de telles colonies hivernales dans la grotte aux Fées supérieure de Vallorbe (VD 6), malheureusement hors de portée pour un dénombrement précis. A. Poillet a fait une observation semblable dans une carrière souterraine de la Franche-Comté, où il a trouvé trois colonies denses de 300 individus chacune, en mars 1962. En été, la barbastelle se réfugie volontiers derrière des contrevents; au moment de la misebas, on peut y observer des colonies composées de quelques dizaines de femelles.

\section{Plecotus auritus (Linné) $)^{3}$ )}

Chacun connaît l'oreillard, si facilement reconnaissable à ses immenses pavillons. Mais ceux-ci sont complètement repliés en arrière et cachés par les bras lors du sommeil hivernal; on ne le reconnaît alors qu'a ses longs oreillons qui pointent en avant. C'est une chauve-souris commune partout en Suisse. On l'a déjà observée dans 36 grottes du Jura, des Alpes et du Tessin. En été, elle se trouve exceptionnellement dans les cavernes; elle fréquente les bâtiments, combles, clochers, où ont lieu les mises-bas.

\section{Myotis daubentoni (Kuhl)}

Le murin de Daubenton fait encore partie des espèces qui se rencontrent assez régulièrement sous terre en hiver. On peut citer 14 grottes du Jura dans lesquelles il a été observé, toujours isolé, souvent profondément enfoncé dans une fissure. En été, il fréquente le bord des eaux et les femelles se rassemblent en petites colonies pour accoucher dans des fentes de rocher, un canal d'écoulement débouchant sur un lac, par exemple.

${ }^{3}$ ) Sous ce nom, je comprends aussi, par souci de simplification, l'oreillard méridional, Plecotus austriacus (Fischer), espèce récemment réhabilitée et qui semble présenter les mèmes mœurs que l'oreillard ordinaire. 
Pour le non-spécialiste, il n'est pas facile d'identifier cette espèce par rapport aux autres murins de taille semblable, comme Myotis emarginatus et M.nattereri. Le caractère le plus frappant concerne les pieds, qui sont très grands chez le murin de Daubenton.

\section{Myotis emarginatus (Geoffroy)}

Le murin à oreilles échancrées est exceptionnel en Suisse et l'on peut compter sur les doigts les individus observés: il y en a exactement 7, plus 4 à l'état d'ossements. Il n'en est pas de mème en France où il constitue l'une des espèces les plus régulièrement trouvées, et souvent en grand nombre, dans les grottes (Ouest et Bassin Parisien, en particulier).

On connait actuellement 9 lieux de trouvailles en Suisse, dont 6 sont des grottes: Milchlöchli (SO 3), Nidlenloch (SO 1, ossements d'un individu), grotte du Lierre (NE 20), grotte de Moron-Ouest (NE 10), grotte aux Amblytèles (NE 27, ossements de 3 individus) et grotte du Bois du Corps de Garde (VD 24). Il a été trouvé hors de grottes à Valavran (Genève), au Col de Balme (Valais) et à Chiasso (planche 2).

En France, Myotis emarginatus est souvent associé au grand rhinolophe et au rhinolophe euryale. Les mises-bas ont lieu soit dans des bâtiments soit dans des grottes. Les biotopes d'hibernation sont constitués par des cavernes naturelles ou artificielles.

\section{Myotis bechsteini (Kuhl)}

Avec le murin de Bechstein, nous abordons l'examen des espèces rencontrées occasionnellement dans nos grottes. Il s'agit d'une chauvesouris arboricole, rare partout, signalée seulement en une douzaine de points sur l'ensemble de la Suisse.

De taille moyenne, elle est facilement reconnaissable à ses très grandes oreilles qui lui confèrent la silhouette de l'oreillard; mais chez ce dernier les deux pavillons sont réunis à la base sur le sommet de la tête, alors que chez le murin de Bechstein les oreilles sont nettement séparées, comme chez tous les autres murins.

Un individu vivant a été observé dans la grotte de la Grande Poule (VD 12) par P. Strinati, et des ossements appartenant à 6 individus ont été exhumés des grottes des Roches Bornel (NE 42), de Pertuis (NE 26, 2 spécimens) et la grotte aux Amblytèles (NE 27, 3 spécimens). Hors de grottes, Myotis bechsteini n'est connu en Suisse que par des trouvailles isolées ${ }^{4}$ ), sauf deux petites colonies estivales dans des

4) Comano (Lugano), Bâle, Neue Welt (Bâle), Oberbipp (Berne), Erschwil, Rosegg et Kestenholz (Soleure). 
nichoirs artificiels à Commugny (Vaud, par J. Steffen) et à Bachs (Zurich, par M.Furrer), respectivement de 22 et 18 individus (planche 2).

\section{Myotis nattereri (Kuhl)}

Le murin de Natterer est aussi une chauve-souris arboricole, dont les mœurs sont semblables à celles de l'espèce précédente. Il est toutefois un peu plus fréquent en Suisse et on l'a trouvé isolément dans 4 grottes: de la Faille (BE 2), de Pertuis (NE 26), aux Amblytèles (NE 27, ossements de 6 individus), du Poteux (VS 2).

La plupart des trouvailles hors de grottes concernent des spécimens isolés ${ }^{5}$ ), sauf deux petites colonies estivales dans un arbre creux à Troinex (Genève) et dans un nichoir artificiel à Commugny (Vaud) (planche 2).

\section{Eptesicus serotinus (Schreber)}

La sérotine est la plus grande chauve-souris, après le grand murin et le grand rhinolophe, qui se rencontre occasionellement sous terre. Elle est assez rare dans l'ensemble de la Suisse et ne se trouve guère qu'en plaine.

Les quelques trouvailles dans les grottes à signaler concernent des individus isolés observés pendant l'hiver: grottes de la Faille (BE 2), de l'Ours (NE 23), de Ver (NE 15), aux Amblytèles (NE 27) et aux Fées supérieure de Vallorbe (VD 6). En été, la sérotine se réfugie volontiers dans les combles ou les vieux bâtiments où a lieu la mise-bas.

\section{Pipistrellus pipistrellus (Schreber)}

La pipistrelle ordinaire est la plus petite espèce, et probablement la plus commune, de Suisse. Mais, comme elle a avant tout des mœurs arboricoles, elle ne se trouve qu'exceptionnellement dans le domaine souterrain, et toujours pour l'hibernation: grotte de Ver (NE 15) et grotte du Poteux (VS 2). Les restes osseux d'un individu ont en outre été trouvés dans la Chorbalm (BE 39). Ses refuges hivernaux sont les trous des arbres et les interstices des bâtisses.

\section{[Nyctalus noctula (Schreber)}

Je ne cite que pour mémoire la trouvaille exceptionnelle de cette espèce, habituellement arboricole (parfois aussi dans des bâtiments),

$\left.{ }^{5}\right)$ Mont Vully (Fribourg), Steinhof (Soleure), St-Gall, Berg (St-Gall), canton de Thurgovie, Lucerne, Andermatt et vallée d'Urseren (Uri), Rabius et Haute-Engadine (Grisons), Simplon et col de Bretolet (Valais). 
dans la Glitzersteinhöhle (SO 2). Un spécimen a été capturé en 1945, il est déposé au muséum de Bâle.]

\section{Eptesicus nilssoni (Keyserling et Blasius)}

La sérotine boréale atteint au Jura et aux Alpes suisses les limites occidentale et méridionale de son aire de répartition. Comme la sérotine ordinaire, c'est une espèce arboricole qui ne se rencontre à l'occasion dans le domaine souterrain que pendant la mauvaise saison. Mais à l'encontre de celle-ci, elle habite seulement les régions montagneuses. Quatre grottes du Jura ont livré jusqu'à présent 14 Eptesicus nilssoni: grottes de l'Echelette (BE 7), de la Faille (BE 2), aux Fées supérieure et inférieure de Vallorbe (VD 6 et VD 7).

En dehors du domaine souterrain et durant la belle saison, la sérotine boréale a été trouvée à Pontresina, val Cluoza, Maloja (Grisons), Uetliberg (Zurich), Grenchenberg (Soleure), Lucerne (?), Loèche-les-Bains et col de Bretolet (Valais) (planche 1).

On ne connaît pas de colonies ou de lieux de mise-bas en Suisse.

\section{Myotis capaccinii (Bonaparte)}

Le murin de Capaccini, très voisin de celui de Daubenton, est une espèce méridionale n'atteignant la Suisse qu'au Tessin. Sur le pourtour de la Méditérranée, il fréquente volontiers les grottes et les souterrains artificiels. La seule station connue au Tessin, d'ailleurs disparue, est la galleria di San Martino (près de Lugano), un tunnel désaffecté dans lequel une colonie importante a été découverte au début de ce siècle. Il a encore été trouvé dans les environs immédiats, au promontoire de San Martino, en 1909.

Ses mœurs sont comparables à celles du murin de Daubenton.

Cette rapide revue de nos chauves-souris cavernicoles permet de constater que si le nombre des espèces rencontrées sous terre est déjà considérable, il reste encore bien des points importants à préciser dans le comportement, les déplacements saisonniers éventuels et la répartition géographique de plusieurs d'entre-elles.

D'autre part, j'espère avoir attiré l'attention des spéléologues sur le rôle important joué par les chauves-souris en tant qu'élément direct et indirect de la faune cavernicole.

\section{RÉSUMÉ}

Les chauves-souris, bien connues des spéléologues, jouent un rôle important dans le peuplement animal des cavernes: d'une façon indirecte par l'apport de guano, source de nourriture pour de nombreux animaux cavernicoles, d'une façon directe par leur présence elle-même plus ou moins régulière. 
On trouve 26 espèces de chauves-souris en Suisse, dont 15 se rencontrent dans les grottes. Seul, Miniopterus schreibersi peut être considéré comme un vrai cavernicole.

La répartition précise des espèces rares est indiquée dans le texte et sur des cartes, aussi pour les trouvailles faites hors de grottes.

\section{SUMMARY}

Bats, familiar to speologists, play an important part in animal ecology in caves. Indirectly by their guano, they provide a source of food for numerous cave-dwelling animals and directly, by their own more or less constant presence.

26 species of bats are known from Switzerland, 15 of which occur in caves. Miniopterus schreibersi is considered the only true cave-dweller.

The exact distribution of the rare species, including those occuring outside caves, is found in the text and is also indicated on the accompanying maps.

\section{B I B LI O GRAP H I E}

Aellen, V. (1949) - Les chauves-souris du Jura neuchâtelois et leurs migrations. Bull. Soc. neuchâteloise Sci. nat. 72: 23-90.

- (1952) - Baguement des chauves-souris dans le Jura suisse. Ornithol. Beob. 49: 8-17.

- (1960 - Notes sur les puces des chauves-souris, principalement de la Suisse (Siphonaptera: Ischnopsyllidae). Bull. Soc. neuchâteloise Sci. nat. 83: 41-61.

- (1962) - Le baguement des chauves-souris au col de Bretolet (Valais). Arch. Sci. Genève 14: 365-392.

- (1963) - Les nyctéribiidés de la Suisse, diptères parasites de chauvessouris. Bull. Soc. neuchateloise Sci. nat. 86: 143-154.

Aellen, V., et Strinati, P. (1962) - Nouveaux matériaux pour une faune cavernicole de la Suisse. Rev. suisse Zool. 69: 25-66.

Anon (1957) - Fruchtbare Zusammenarbeit. Stalactite (Org. Soc. suisse Spéléol.) 7: 126-127.

Bernasconi, R. (1956) - Notes sur quelques nouvelles grottes au Tessin méridional. Stalactite (Org. Soc. suisse Spéléol.) 6: 89-94.

Cotт, G. (1959) - Le grotte del Ticino II. Note biologiche I. Parte II. Boll. Soc. ticinese Sci. nat. 53:43-74.

Desfayes, M. (1954) - Les chauves-souris de la grotte du Poteux (Saillon). Bull. Murith. Sion 71: 46-51.

Furrer, M. (1957) - Ökologische und systematische Übersicht über die Chiropterenfauna der Schweiz. Laupen (Bern), 1-87.

Gigon, R., et Aellen, V. (1960) - Contribution à la spéléologie dubassin suisse du Doubs. Stalactite (Org. Soc. suisse Spéléol.) 4: 79-123.

Hainard, R. (1961) - Mammifères sauvages d'Europe I. Insectivores, Chéiroptères, Carnivores. Neuchâtel: 1-322.

JÉquier, J. P. (1961) - Les restes osseux de la grotte aux Amblytèles. Cavernes (Bull: Spéléo-Cl. Mont. neuchâteloises) 5: 1-4.

Mislin, H. (1945) - Zur Biologie der Chiroptera, III. Erste Ergebnisse der Fledermausberingung im Jura (Beobachtungen in den Winterquartieren 1940/45). Res. suisse Zool. 52: 371-376. 
Strinati, P. (1953) - Capture de Myotis bechsteinii (Kuhl) dans une grotte du Jura vaudois. Stalactite (Org. Soc. suisse Spéléol.) 3 (5): 14.

\section{EXPLICATIONS DES PLANGHES 67(1)-68(2)}

\section{PLANCHE 67 (1)}

Répartition en Suisse de Miniopterus schreibersi (Kuhl) et de Eptesicus nilssoni (Keyserling et Blasius).

\section{PLANCHE $68(2)$}

Répartition en Suisse de Myotis nattereri (Kuhl), Myotis emarginatus (Geoffroy) et Myotis bechsteini (Kuhl).

\section{PLANCHE 69 (3)}

Photos V. Aellen de gauche à droite et de haut en bas: Miniopterus schreibersi (Kuhl), grotte du Chemin de Fer, Jura neuchâtelois. Rhinolophus hipposideros (Bechstein), grotte La Catalanna, Haute-Savoie. Plecotus auritus (Linné), grotte de Vers-chez-le-Brandt, Jura neuchâtelois. Myotis bechsteini (Kuhl), Commugny, Vaud. Myotis nattereri (Kuhl), grotte de la Faille, Jura bernois. Eptesicus nilssoni (Keyserling et Blasius), grotte aux Fées de Vallorbe, Jura vaudois. 


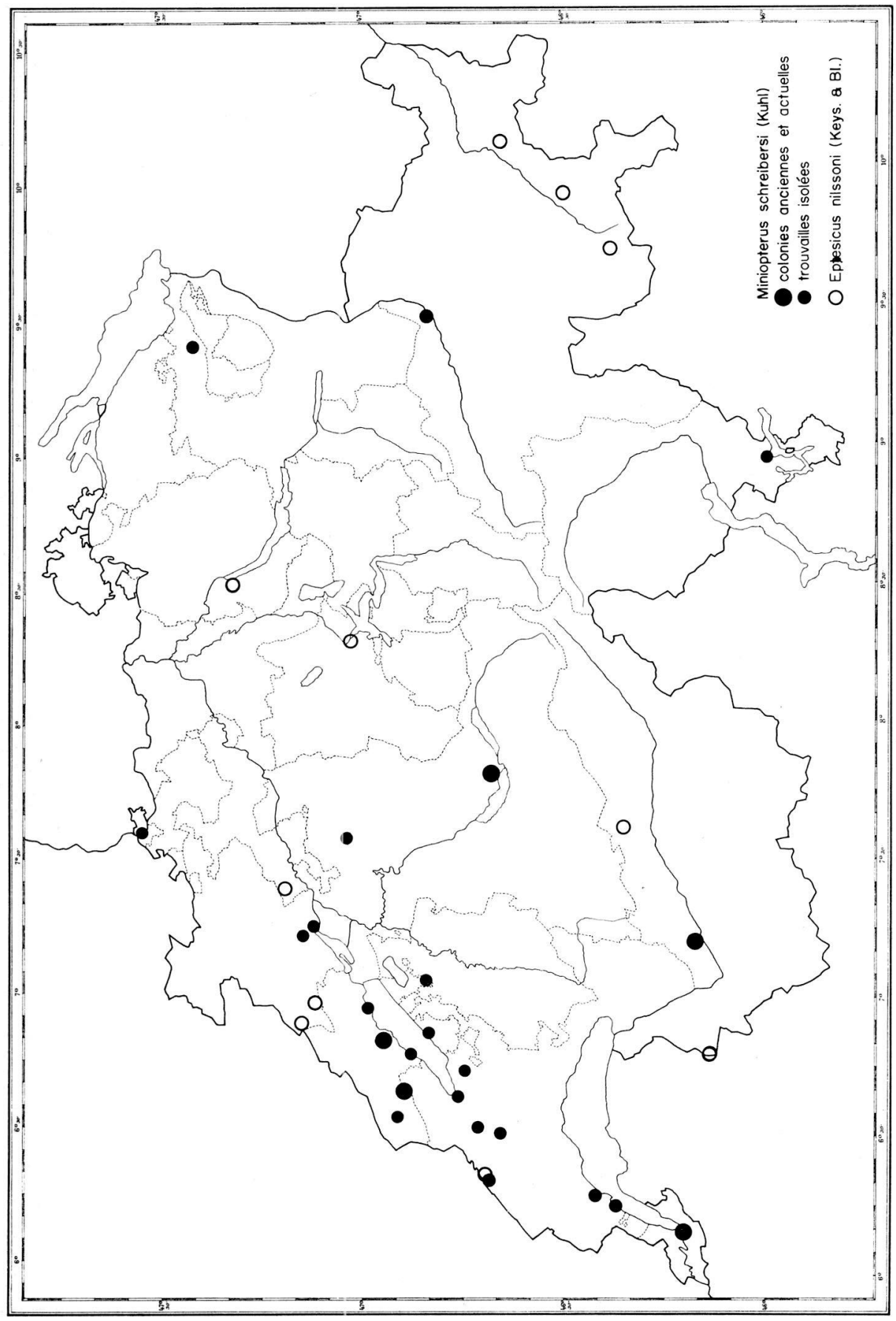




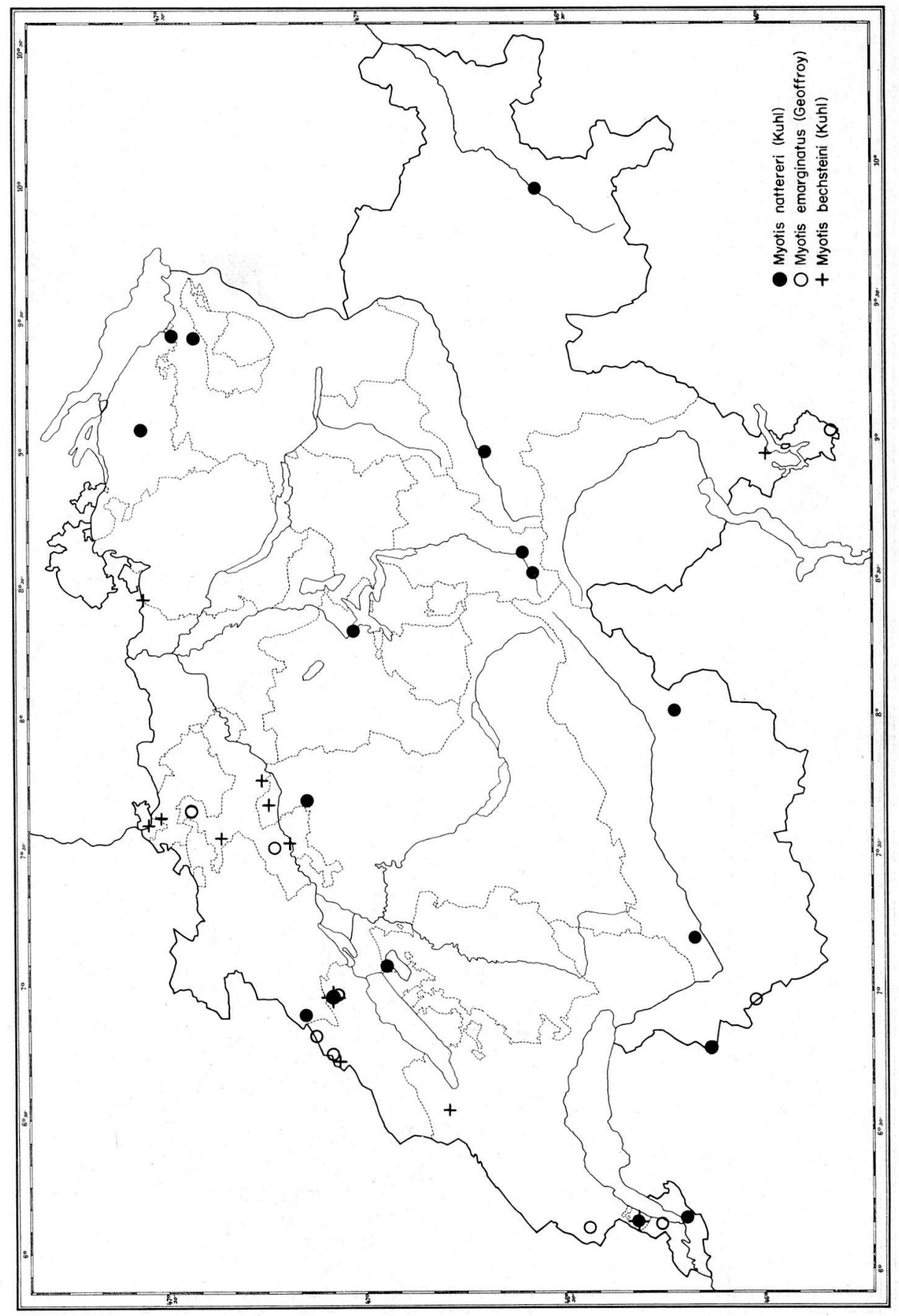



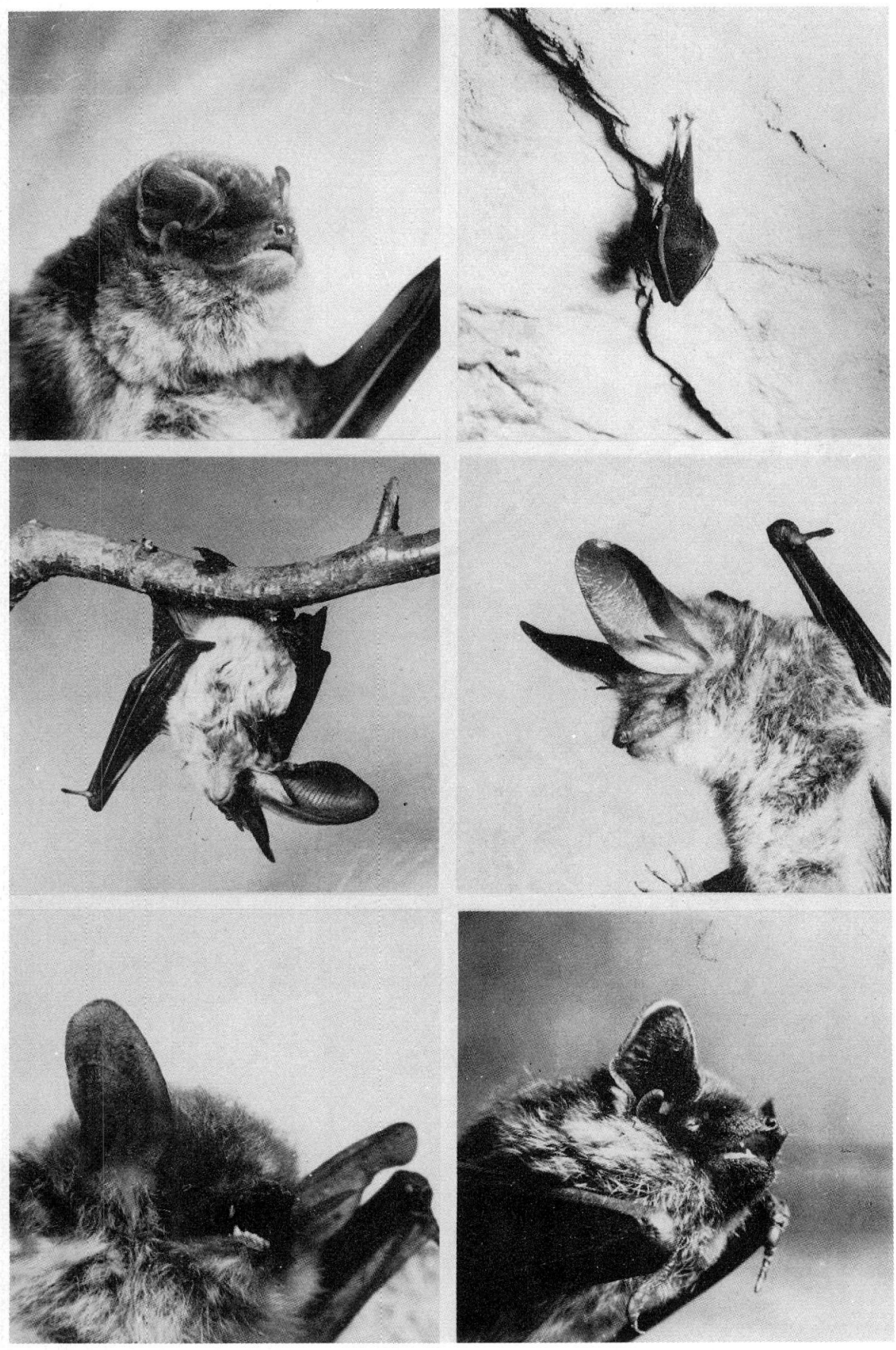